\title{
The Power of Change-Point Test for Two-Phase Regression
}

\author{
To Van Ban, Nguyen Thi Quyen \\ Department of Mathematics, Haiphong University, Haiphong, Vietnam \\ Email: quyentthp@yahoo.com
}

Received 3 September 2014; revised 28 September 2014; accepted 12 October 2014

Copyright (C) 2014 by authors and Scientific Research Publishing Inc.

This work is licensed under the Creative Commons Attribution International License (CC BY). http://creativecommons.org/licenses/by/4.0/

c) (i) Open Access

\begin{abstract}
In this paper, the roughness of the model function to the basis functions and its properties have been considered. We also consider some conditions to take the limit of the roughness when the observations are i.i.d. An explicit formula to calculate the power of change-point test for the two phases regression through the roughness was obtained.
\end{abstract}

\section{Keywords}

\section{Change-Point Test, The Power, The Roughness, Random Design}

\section{Introduction}

Many authors have used the likelihood ratio to study the change-point problem (see [1] [2]). Worsley, K.J. [3] gave exact approximate bounds for the null distributions of likelihood ratio statistics in two case of known and unknown variance. Simulation study results indicated that the approximation of his upper bound is very good for the small sample size, but the study does not support the case of large one. Koul and H.L, Qian. L. [4] studied the change-point by the maximum likelihood and random design. In the case of known variance, Jaruskova, D. [5] derived an asymptotic distribution of log-likelihood type ratio to detect a change-point from a known (or unknown) constant state to a trend state. Aue A., Horvath, L., Huskova, M. and Kokoszka, P. [1] studied the limit distribution of the trimmed version of the likelihood ratio, from which they received the test statistic to detect a change-point for the polynomial regressions. Researchers have used to take simulation studies on the various scenarios of the parameters of alternative hypothesis to find the power of a test. They have found that it depends on the sample size, variance of error and the behavior of the model function under alternative. For two phases' regression, Lehmann, E.L. and Romano, J.P. [6] gave a formula to calculate the power of change-point through the noncentral F-distribution.

In this paper, the behavior of the model function under alternative is quantified by the roughness that is used 
to calculate the power of tests. The present paper is organized in the following way. In Section 2, we give a definition of the roughness of the model function and show some its properties; it is possible to take the limit of the roughness when the sequence of designs converges weakly to a limit design as well as designs are random. In Section 3, we present an explicit formula to calculate the noncentrality parameter of F-test in [6] through the roughness, and then the power of change-point test and some of its limits are considered.

\section{The Roughness of the Model Function}

In what follows, we will denote the unit matrix of size $n$ by $\boldsymbol{I}_{n}$, the matrix having elements $a_{i j}$ in the $i$-th row and $j$-th column by $\left(a_{i j}\right)$, the transpose of a matrix $\boldsymbol{A}$ by $\boldsymbol{A}^{\prime}$, the Euclidean norm of a vector and the maximum norm of a matrix by $\|$.$\| . For a distribution function F(x)$ whose support belongs to $I$, we will denote the Lebesgue-Stieltjes measure associated with $F(x)$ by $(\mathrm{d} F)$, and choose $\langle h, g\rangle=\int_{I} h(x) g(x) \mathrm{d} F(x)$ as the inner product in $L_{2}(F)$.

To approximate the function $f(x)$ by a given system of functions $\left\{z_{1}(x), \cdots, z_{p}(x)\right\}$ at the given points $x_{i} \in I, i=1, \cdots, n$, we consider the model

$$
\begin{array}{r}
f\left(x_{i}\right)=\alpha_{1} z_{1}\left(x_{i}\right)+\cdots+\alpha_{p} z_{p}\left(x_{i}\right)+\gamma_{i}, i=1, \cdots, n . \\
\text { Let } \mathbf{z}(x)=\left(\begin{array}{c}
z_{1}(x) \\
\vdots \\
z_{p}(x)
\end{array}\right), \boldsymbol{Z}(x)=\left(\begin{array}{c}
\mathbf{z}^{\prime}\left(x_{1}\right) \\
\vdots \\
\mathbf{z}^{\prime}\left(x_{n}\right)
\end{array}\right), \boldsymbol{Y}=\left(\begin{array}{c}
f\left(x_{1}\right) \\
\vdots \\
f\left(x_{n}\right)
\end{array}\right), \boldsymbol{\alpha}=\left(\begin{array}{c}
\alpha_{1} \\
\vdots \\
\alpha_{p}
\end{array}\right), \boldsymbol{\gamma}=\left(\begin{array}{c}
\gamma_{1} \\
\vdots \\
\gamma_{n}
\end{array}\right) .
\end{array}
$$

In matrix notation, (1) is written as

$$
\boldsymbol{Y}=\mathbf{Z} \boldsymbol{\alpha}+\boldsymbol{\gamma} .
$$

We always assume that $\operatorname{Rank}(\boldsymbol{Z})=p$, then the estimate for $\boldsymbol{\alpha}$ that minimizes the mean square error $\frac{1}{n} \sum_{i=1}^{n}\left(f\left(x_{i}\right)-\mathbf{z}^{\prime}\left(x_{i}\right) \alpha\right)^{2}$ is

$$
\hat{\alpha}=\left(Z^{\prime} Z\right)^{-1} Z^{\prime} Y
$$

and the estimate for the error of the model (1) is

$$
S^{2}=\frac{1}{n} \sum_{i=1}^{n}\left(f\left(x_{i}\right)-\mathbf{Z}^{\prime}\left(x_{i}\right) \hat{\alpha}\right)^{2}=\frac{1}{n}\left\|\left(\boldsymbol{I}_{n}-\mathbf{Z}\left(\mathbf{Z}^{\prime} \mathbf{Z}\right)^{-1} \mathbf{Z}^{\prime}\right) \boldsymbol{Y}\right\|^{2} .
$$

We call this value the roughness of the function $f(x)$ to the system of functions $\left\{z_{1}(x), \cdots, z_{p}(x)\right\}$ based on the design $\left\{x_{1}, \cdots, x_{n}\right\}$ and denote it by $S^{2}\left(f, \mathbf{z},\left\{x_{i}\right\}_{n}\right)$. In the case of a linear trend where $p=2, z_{1}(x)=1$, $z_{2}(x)=x, \quad S^{2}$ shows the nonlinearity of the curve $y=f(x)$ based on observations at $x_{1}, \cdots, x_{n}$.

To study limits cases as well as other purposes, we call a distribution function $F(x)$, whose support belongs to $I$ a (generalized) design on $I$. A design $F(x)$ is called to be adapted to a system of functions $\left\{z_{1}(x), \cdots, z_{p}(x), x \in I\right\}$ if its support belongs to $I$ so that the matrix $\left\langle\mathbf{z}, \mathbf{z}^{\prime}\right\rangle_{F}=\left(\left\langle z_{i}, z_{j}\right\rangle_{F}\right)$ is invertible. In this paper, the used designs are assumed to be adapted to the system $\left\{z_{1}(x), \cdots, z_{p}(x), x \in I\right\}$.

To continue, we will establish some assumptions:

(A1) The model function $f(x), x \in I$ is measurable and bounded,

(A2) Trend functions $z_{1}(x), \cdots, z_{p}(x), x \in I$ are linearly independent and continuous.

Now suppose that (A1) and (A2) hold, we approximate the function $f(x)$ to $\left\{z_{1}(x), \cdots, z_{p}(x)\right\}$ in the equation:

$$
f(x)=\alpha_{1} z_{1}(x)+\cdots+\alpha_{p} z_{p}(x)+\gamma(x) .
$$

The estimate for the parameter vector $\boldsymbol{\alpha}=\left(\alpha_{1}, \cdots, \alpha_{p}\right)^{\prime}$ that minimizes the weighted mean square error $\int_{I}\left(f(x)-\mathbf{z}^{\prime}(x) \alpha\right)^{2} \mathrm{~d} F(x)$ is 


$$
\hat{\alpha}=\left(\hat{\alpha}_{1}, \cdots, \hat{\alpha}_{p}\right)^{\prime}=\left(\left\langle\mathbf{z}, \mathbf{z}^{\prime}\right\rangle_{F}\right)^{-1}\langle\mathbf{z}, f\rangle_{F},
$$

where $\langle\mathbf{z}, f\rangle_{F}=\left(\left\langle z_{1}, f\right\rangle_{F}, \cdots,\left\langle z_{p}, f\right\rangle_{F}\right)^{\prime}$. Hence, the estimate for the error of the model (5) is

$$
S^{2}=\int_{I}\left(f(x)-z^{\prime}(x) \hat{\alpha}\right)^{2} \mathrm{~d} F(x) .
$$

We also call this value the roughness of the function $f(x)$ to the system of trend functions $\left\{z_{1}(x), \cdots, z_{p}(x)\right\}$ based on the design $F(x)$ and denote it by $S^{2}(f, z, F)$. It is easily seen that each discrete design is a generalized design, thus (3), (4) is a special case of (6), (7), respectively.

According to [2], to evaluate the roughness of the model function based on polynomial trend functions $\left\{1, x, \cdots, x^{n}\right\}$, by using the linear transformation of independent variables, instead of observing on the arbitrary interval $[a, b]$, one can observe on the standard interval $[0,1]$. Then, from now on, the model functions defined on $[0,1]$ are considered only.

The following theorem in [7] shows the conditions for occurring the convergence of the estimated parameters and the roughness.

Theorem 1. Assume that conditions (A1), (A2) are satisfied where $I=[0,1]$. Let $F_{n}(x), n=1,2, \cdots, F(x)$ be designs, and $\hat{\alpha}_{n}, S^{2}\left(f, \mathbf{z}, F_{n}\right), \hat{\alpha}, S^{2}(f, \mathbf{z}, F)$ be the estimates of the coefficients and the roughness of the model function defined by (6) and (7), respectively. Assume that $F_{n}(x)$ converges weakly to $F(x)$ having Lebesgue-Stieltjes $(\mathrm{d} F)$-measure. If $D_{f}$ is the set of discontinuities of $f(x)$, such that $(\mathrm{d} F)\left(D_{f}\right)=0$ then:

1) $\lim \hat{\alpha}_{n}=\hat{\alpha}$,

2) $\lim _{n \rightarrow \infty} S^{2}\left(f, \mathbf{z}, F_{n}\right)=S^{2}(f, \mathbf{z}, F)$.

Now, we consider the model (1) where the observations $X_{1}, X_{2}, \cdots$ are i.i.d. with the distribution function $F(x)$ having support on $I$. The roughness of $f(x)$ is calculated by (4), in which $x_{i}$ is replaced by $X_{i}$ :

$$
S_{n}^{2}\left(f, \mathbf{z},\left(X_{i}\right)\right)=\frac{1}{n}\left\|\left(\boldsymbol{I}_{n}-\boldsymbol{Z}_{n}\left(\boldsymbol{Z}_{n}^{\prime} \boldsymbol{Z}_{n}\right)^{-1} \mathbf{Z}_{n}^{\prime}\right) \boldsymbol{f}_{n}\right\|^{2},
$$

where

$$
\begin{gathered}
\mathbf{z}\left(X_{i}\right)=\left(z_{1}\left(X_{i}\right), \cdots, z_{p}\left(X_{i}\right)\right), \quad i=1, \cdots, p, \\
\mathbf{Z}_{n}=\left(\mathbf{z}^{\prime}\left(X_{1}\right), \cdots, \mathbf{z}^{\prime}\left(X_{n}\right)\right)^{\prime}, \quad \boldsymbol{f}_{n}=\left(f\left(X_{1}\right), \cdots, f\left(X_{n}\right)\right)^{\prime} .
\end{gathered}
$$

Theorem 2. Suppose that (A1) and (A2) hold for $I=[0,1]$ and

1) $X_{0}=X, X_{1}, X_{2}, \cdots$ are independent random variables with the common distribution function $F(x)$ having support on $I$,

2) $\operatorname{Rank}\left(\mathbf{Z}_{n}^{\prime} \mathbf{Z}_{n}\right)=\operatorname{Rank}\left(E\left(z_{i}(X) z_{j}(X)\right)\right)=p, n=1,2, \cdots($ a.s $)$,

3) The roughness $S_{n}^{2}\left(f, \mathbf{z},\left(X_{i}\right)\right)$ is defined by (8).

Then

$$
\lim _{n \rightarrow \infty} S_{n}^{2}\left(f, \mathbf{z},\left(X_{i}\right)\right)=S^{2}(f, \mathbf{z}, F(x))(\text { a.s }) .
$$

Proof. We note that $S_{n}^{2}\left(f, \mathbf{z},\left(X_{i}\right)\right)$ is the sample variance of the model

$$
f\left(X_{i}\right)=\alpha_{1} z_{1}\left(X_{i}\right)+\cdots+\alpha_{p} z_{p}\left(X_{i}\right)+\gamma_{i}, \quad i=1, \cdots, n .
$$

Let $\hat{\alpha}_{n}$ be the least-square estimate of $\alpha$ bases on $n$ observations, we get

$$
\begin{gathered}
\hat{\alpha}_{n}=\left(\hat{\alpha}_{n 1}, \cdots, \hat{\alpha}_{n p}\right)^{\prime}=\left(\mathbf{Z}_{n}^{\prime} \mathbf{Z}_{n}\right)^{-1} \mathbf{Z}_{n}^{\prime} \boldsymbol{f}_{n}=\left(\frac{1}{n} \mathbf{Z}_{n}^{\prime} \mathbf{Z}_{n}\right)^{-1}\left(\frac{1}{n} \mathbf{Z}_{n}^{\prime} \boldsymbol{f}_{n}\right), \\
S_{n}^{2}\left(f, \mathbf{z},\left(X_{i}\right)\right)=\frac{1}{n} \sum_{i=1}^{n}\left(f\left(X_{i}\right)-\left(\hat{\alpha}_{n 1} Z_{1}\left(X_{i}\right)+\cdots+\hat{\alpha}_{n p} Z_{p}\left(X_{i}\right)\right)\right)^{2} .
\end{gathered}
$$


Because $\left\{z_{i}\left(X_{t}\right) z_{j}\left(X_{t}\right), t=0,1,2, \cdots\right\}$ is a sequence of i.i.d. variables which have finite variance then by the strong law of large numbers,

$$
\lim _{n \rightarrow \infty} \frac{1}{n} Z_{n i}^{\prime} \boldsymbol{Z}_{n j}=\lim _{n \rightarrow \infty} \frac{1}{n} \sum_{t=1}^{n} z_{i}\left(X_{t}\right) z_{j}\left(X_{t}\right)=E\left(z_{i}(X) z_{j}(X)\right)(\text { a.s }) .
$$

Then, according to the assumption 2),

$$
\lim _{n \rightarrow \infty} \operatorname{det}\left(\frac{1}{n} \mathbf{Z}_{n}^{\prime} \mathbf{Z}_{n}\right)=\operatorname{det}\left(\left\langle\mathbf{z}, \mathbf{z}^{\prime}\right\rangle_{F}\right) \neq 0(\text { a.s }),
$$

which follows that elements of the matrix $\left(\frac{1}{n} Z_{n}^{\prime} Z_{n}\right)^{-1}$ converge (a.s) to corresponding elements of the matrix $\left(E\left(z_{i}(X) z_{j}(X)\right)\right)^{-1}$.

Similar arguments yield

$$
\lim _{n \rightarrow \infty} \frac{1}{n} Z_{n}^{\prime} \boldsymbol{f}_{n}=\left(E\left(z_{1}(X) f(X)\right), \cdots, E\left(z_{p}(X) f(X)\right)\right)^{\prime} \quad \text { (a.s). }
$$

Consequently, we obtain the limit

$$
\begin{aligned}
\lim _{n \rightarrow \infty} \hat{\boldsymbol{\alpha}}_{n} & =\lim _{n \rightarrow \infty}\left(\frac{1}{n} \boldsymbol{Z}_{n}^{\prime} \mathbf{Z}_{n}\right)^{-1}\left(\frac{1}{n} \boldsymbol{Z}_{n}^{\prime} \boldsymbol{f}_{n}\right) \\
& =\left(E\left(z_{i}(X) z_{j}(X)\right)\right)^{-1}\left(\begin{array}{c}
E\left(z_{1}(X) f(X)\right) \\
\vdots \\
E\left(z_{p}(X) f(X)\right)
\end{array}\right)=\hat{\boldsymbol{\alpha}}=\left(\begin{array}{c}
\hat{\alpha}_{1} \\
\vdots \\
\hat{\alpha}_{p}
\end{array}\right)(\text { a.s }) .
\end{aligned}
$$

Note that $\hat{\alpha}$ is not random and the roughness can be expressed by

$$
S_{n}^{2}\left(f, \mathbf{z},\left(X_{i}\right)\right)=\frac{1}{n} \sum_{i=1}^{n}\left(f\left(X_{i}\right)-\left(\hat{\alpha}_{n 1} z_{1}\left(X_{i}\right)+\cdots+\hat{\alpha}_{n p} z_{p}\left(X_{i}\right)\right)\right)^{2}=\frac{1}{n} \sum_{i=1}^{n}\left(A_{i}+B_{i}\right)^{2},
$$

where

$$
\begin{gathered}
A_{i}=f\left(X_{i}\right)-\left(\hat{\alpha}_{1} z_{1}\left(X_{i}\right)+\cdots+\hat{\alpha}_{p} z_{p}\left(X_{i}\right)\right), \\
B_{i}=\left(\hat{\alpha}_{1}-\hat{\alpha}_{n 1}\right) z_{1}\left(X_{i}\right)+\cdots+\left(\hat{\alpha}_{p}-\hat{\alpha}_{n p}\right) z_{p}\left(X_{i}\right) .
\end{gathered}
$$

Because $\left\{A_{i}\right\}$ are i.i.d. and bounded then according to the central limit theorem,

$$
\begin{aligned}
& \frac{1}{n} \sum_{i=1}^{n} A_{i}^{2} \rightarrow E\left(f(X)-\left(\hat{\alpha}_{1} z_{1}(X)+\cdots+\hat{\alpha}_{p} z_{p}(X)\right)\right)^{2} \\
& =\int_{I}\left(f(x)-\left(\hat{\alpha}_{1} z_{1}(x)+\cdots+\hat{\alpha}_{p} z_{p}(x)\right)\right)^{2} \mathrm{~d} F(x)(n \rightarrow \infty)(\text { a.s }) .
\end{aligned}
$$

Inasmuch as $\hat{\alpha}=\left(\hat{\alpha}_{1}, \cdots, \hat{\alpha}_{p}\right)^{\prime}$ satisfies (9), it can be calculated by (6). Hence, the right side of (10) is $S^{2}(f, z, F(x))$.

Again, according to the central limit theorem and (9),

$$
\left|\frac{1}{n} \sum_{i=1}^{n} B_{i}^{2}\right| \leq \max _{i=1, \cdots, p}\left|\hat{\alpha}_{i}-\hat{\alpha}_{n i}\right|^{2} \times \frac{1}{n} \sum_{i=1}^{n}\left(\left|z_{1}\left(X_{i}\right)\right|+\cdots+\left|z_{p}\left(X_{i}\right)\right|\right)^{2} \rightarrow 0 \quad(n \rightarrow \infty) \quad \text { (a.s). }
$$

Combining the above with the fact that $\left(A_{i}+B_{i}\right)^{2} \leq 2\left(A_{i}^{2}+B_{i}^{2}\right)$ we obtain

$$
\lim _{n \rightarrow \infty} S_{n}^{2}\left(f, \mathbf{z},\left(X_{i}\right)\right)=S^{2}(f, \mathbf{z}, F(x)) \quad(a . s) .
$$


This completes the proof of the theorem.

\section{Applications to the Change-Point Test}

Suppose that the model function is defined as:

$$
f(x)=\left\{\begin{array}{l}
\beta_{11} z_{1}(x)+\cdots+\beta_{1 p} z_{p}(x) \text { if } a \leq x \leq x^{*}, \\
\beta_{21} z_{1}(x)+\cdots+\beta_{2 p} z_{p}(x) \text { if } x^{*}<x \leq b,
\end{array}\right.
$$

where $z_{i}(x)$ are known functions, $\beta_{i j}$ are unknown parameters. Observations $\left\{x_{1}, x_{2}, \cdots, x_{n}\right\}$ belong to the closed interval $I=[a, b]$, without the loss of generality, we can assume $x_{1} \leq x_{2} \leq \cdots \leq x_{n}$, some $x_{i}$ can be identical. Suppose that a change-point happened at a some time $k^{*}$, the model is written:

$$
y_{i}= \begin{cases}\beta_{11} z_{1}\left(x_{i}\right)+\cdots+\beta_{1 p} z_{p}\left(x_{i}\right)+\varepsilon_{i} & \text { if } i=1, \cdots, k^{*}, \\ \beta_{21} z_{1}\left(x_{i}\right)+\cdots+\beta_{2 p} z_{p}\left(x_{i}\right)+\varepsilon_{i} & \text { if } i=k^{*}+1, \cdots, n,\end{cases}
$$

where $\left\{\varepsilon_{i}\right\}$ is a sequence of i.i.d. variables $N\left(0, \sigma^{2}\right)$ with the unknown common variance $\sigma^{2}$.

Let

$$
\begin{aligned}
& \mathbf{z}(x)=\left(\begin{array}{c}
z_{1}(x) \\
\vdots \\
z_{p}(x)
\end{array}\right), \boldsymbol{Z}_{1}=\left(\begin{array}{c}
\mathbf{z}^{\prime}\left(x_{1}\right) \\
\vdots \\
\mathbf{z}^{\prime}\left(x_{k^{*}}\right)
\end{array}\right), \boldsymbol{Z}_{2}=\left(\begin{array}{c}
\mathbf{z}^{\prime}\left(x_{k^{*}+1}\right) \\
\vdots \\
\mathbf{z}^{\prime}\left(x_{n}\right)
\end{array}\right), \boldsymbol{Z}=\left(\begin{array}{c}
\mathbf{Z}_{1} \\
\boldsymbol{Z}_{2}
\end{array}\right), \boldsymbol{Y}_{1}=\left(\begin{array}{c}
y_{1} \\
\vdots \\
y_{k^{*}}
\end{array}\right), \boldsymbol{Y}_{2}=\left(\begin{array}{c}
y_{k^{*}+1} \\
\vdots \\
y_{n}
\end{array}\right), \\
& \boldsymbol{Y}=\left(\begin{array}{c}
\boldsymbol{Y}_{1} \\
\boldsymbol{Y}_{2}
\end{array}\right), \boldsymbol{f}=\left(\begin{array}{c}
\boldsymbol{Z}_{1} \boldsymbol{\beta}_{1} \\
\boldsymbol{Z}_{2} \boldsymbol{\beta}_{2}
\end{array}\right), \boldsymbol{\varepsilon}_{1}=\left(\begin{array}{c}
\varepsilon_{1} \\
\vdots \\
\varepsilon_{k^{*}}
\end{array}\right), \boldsymbol{\varepsilon}_{2}=\left(\begin{array}{c}
\varepsilon_{k^{*}+1} \\
\vdots \\
\varepsilon_{n}
\end{array}\right), \boldsymbol{\varepsilon}_{1}=\left(\begin{array}{c}
\boldsymbol{\varepsilon}_{11} \\
\boldsymbol{\varepsilon}_{2}
\end{array}\right), \boldsymbol{\beta}_{i}=\left(\begin{array}{c}
\vdots \\
\beta_{i p}
\end{array}\right), i=1,2 .
\end{aligned}
$$

Using matrix notations, the Equation (12) is written as

$$
\boldsymbol{Y}_{i}=\mathbf{Z}_{i} \boldsymbol{\beta}_{i}+\boldsymbol{\varepsilon}_{i}, i=1,2 \quad \text { or } \quad \boldsymbol{Y}=\left(\begin{array}{c}
\boldsymbol{Z}_{1} \boldsymbol{\beta}_{1}+\boldsymbol{\varepsilon}_{1} \\
\boldsymbol{Z}_{2} \boldsymbol{\beta}_{2}+\boldsymbol{\varepsilon}_{2}
\end{array}\right)
$$

We are interested in testing the hypothesis of structural stability against the alternative of a regime switch at a sometime $k^{*}$, that is

$$
H_{0}: \boldsymbol{\beta}_{1}=\boldsymbol{\beta}_{2}=\boldsymbol{\beta} \text { against } H_{1}: \boldsymbol{\beta}_{1} \neq \boldsymbol{\beta}_{2} .
$$

Let $k^{*}$ be known as it was studied in Bischoff and Miller [8]. In addition, we assume that the matrices $\mathbf{Z}_{1}, \mathbf{Z}_{2}, \boldsymbol{Z}$ have full rank: $\operatorname{Rank}\left(\mathbf{Z}_{1}\right)=\operatorname{Rank}\left(\mathbf{Z}_{2}\right)=\operatorname{Rank}(\boldsymbol{Z})=p$ thence $n \geq 2 p$. From that, vector $\boldsymbol{f}$ belongs to a $2 p$-dimensional linear subspace $\Pi_{\Omega}$, and the null hypothesis $H_{0}$ to test that $f$ lies in a $p$ dimensional subspace $\Pi_{\omega}$ of $\Pi_{\Omega}$.

The least-squares estimate of $\boldsymbol{\beta}$ under $H_{0}$ and $\boldsymbol{\beta}_{1}, \boldsymbol{\beta}_{2}$ under $H_{1}$ are $\hat{\boldsymbol{\beta}}=\left(\mathbf{Z}^{\prime} \mathbf{Z}\right)^{-1} \boldsymbol{Z}^{\prime} \boldsymbol{Y}$, $\hat{\boldsymbol{\beta}}_{1}=\left(\boldsymbol{Z}_{1}^{\prime} \boldsymbol{Z}_{1}\right)^{-1} \boldsymbol{Z}_{1}^{\prime} \boldsymbol{Y}_{1}$ and $\hat{\boldsymbol{\beta}}_{2}=\left(\boldsymbol{Z}_{2}^{\prime} \mathbf{Z}_{2}\right)^{-1} \boldsymbol{Z}_{2}^{\prime} \boldsymbol{Y}_{2}$, respectively. Let $\hat{\boldsymbol{Y}}, \hat{\hat{\boldsymbol{Y}}}$ are the orthogonal projections of $\boldsymbol{Y}$ onto $\Pi_{\Omega}$ and $\Pi_{\omega}$ then $\hat{\boldsymbol{Y}}=\left(\begin{array}{c}\boldsymbol{Z}_{1} \boldsymbol{\beta}_{1} \\ \boldsymbol{Z}_{2} \boldsymbol{\beta}_{2}\end{array}\right)$ and $\hat{\hat{\boldsymbol{Y}}}=\boldsymbol{Z} \hat{\boldsymbol{\beta}}$.

We already know that (see Lehmann, E.L. and Romano, J.P. [6]): Under $H_{0}$, the statistics

$$
F=\frac{\left[\|\hat{\boldsymbol{Y}}-\hat{\hat{\boldsymbol{Y}}}\|^{2}-\|\boldsymbol{Y}-\hat{\boldsymbol{Y}}\|^{2}\right] / p}{\|\boldsymbol{Y}-\hat{\boldsymbol{Y}}\|^{2} /(n-2 p)}
$$

will be distributed $F(p, n-2 p)$. Thus, the test rejects the null hypothesis at level $\alpha$ if

$$
F>f_{\alpha}(p, n-2 p),
$$


where $f_{\alpha}(p, n-2 p)$ is the $\alpha$-critical value for $F(p, n-2 p)$, a $F$-distributed random variable with $p$ and $n-2 p$ degrees of freedom. According to [6], by denoting $g(\boldsymbol{X})=\|\boldsymbol{X}-\hat{\hat{\boldsymbol{X}}}\|^{2}-\|\boldsymbol{X}-\hat{\boldsymbol{X}}\|^{2}$, where $\hat{\boldsymbol{X}}, \hat{\hat{\boldsymbol{X}}}$ are orthogonal projections of $\boldsymbol{X}$ onto $\Pi_{\Omega}$ and $\Pi_{\omega}$, respectively, then under $H_{1}$, the statistic $F$ defined by (15) will be noncentral $F$-distribution with $p, n-2 p$ degrees of freedom and noncentrality parameter $\lambda=\frac{1}{\sigma^{2}} g(\boldsymbol{f})$.

We note that $\hat{\boldsymbol{f}}=\boldsymbol{f}$ and $\hat{\hat{f}}=\mathbf{Z}\left(\mathbf{Z}^{\prime} \mathbf{Z}\right)^{-1} \mathbf{Z}^{\prime} \boldsymbol{f}=\mathbf{Z} \hat{\boldsymbol{\alpha}}$, which implies that

$$
\lambda=\frac{\|\boldsymbol{f}-\boldsymbol{Z} \hat{\boldsymbol{\alpha}}\|^{2}}{\sigma^{2}}=\frac{n S^{2}\left(f, \mathbf{z},\left\{x_{i}\right\}_{n}\right)}{\sigma^{2}} .
$$

Now, we call $R_{n}=\frac{S^{2}\left(f, \mathbf{z},\left\{x_{i}\right\}_{n}\right)}{\sigma^{2}}$ and $R=\frac{S^{2}(f, \mathbf{z}, F(x))}{\sigma^{2}}$ the signal-to-noise of the model (11) based on the design $F(x)$ and $\left\{x_{i}\right\}_{n}$, respectively.

Theorem 3. If assumptions (A2), (A3) hold then the power of test (16) is defined by

$$
\text { Pow }=P\left\{F\left(p, n-2 p, n R_{n}\right)>f_{\alpha}(p, n-2 p)\right\} \text {. }
$$

Remark. Theorem 3 shows an explicit formula of the power of change-point test. In the case of $p=2$ and $z_{1}(x)=1, z_{2}(x)=x$, if the model function $f(x)$ is continuous segment, the shift of the slope between the first segment and the last one is $h$, by Theorem 1 in [7], the maximum roughness is obtained if the change-point $x^{*}$ is the midpoint of the observations. With the given common variance $\sigma^{2}$ of the model, the maximum signalto-noise $R$ is obtained at this change-point, thence from Theorem 3, the power is maximum. This fits results of simulation studies in [1].

To increase signal-to-noise ratio, we can decrease the noise or increase the roughness of the model function. When the variance $\sigma^{2}$ is small, we can assert that if the model function has a change-point then this test will find it surely. On the other hand, if the variance is large, the test is poorly.

With the sample size $n$ and design $\left\{x_{i}\right\}_{n}$ if the variance $\sigma^{2}$ decreases to 0 then $R$ increases to $\infty$ and if $\sigma^{2}$ increases to $\infty$, then $R$ decreases to 0 . We have the following corollaries that show the relationship between power and the common variance and the roughness.

Corollary 1. If the assumptions in Theorem 3 are satisfied then the following limits hold:

1) $\lim _{\sigma \rightarrow 0}$ Pow $=1$,

2) $\lim _{\sigma \rightarrow \infty}$ Pow $=\alpha$.

Limits of the powers are obtained by the following corollary.

Corollary 2. 1) With the same conditions as in Theorem 3, assume that $R_{n} \geq R_{0}$ for every $n \geq N_{0} \in \mathbb{N}$ and some $R_{0}>0$ then $\lim _{n \rightarrow \infty}$ Pow $_{n}=1$.

2) Furthermore, if the model function $f(x)$ and a sequence of designs $F_{n}(x)$ satisfies the conditions of Theorem 1, then $\lim _{n \rightarrow \infty} \operatorname{Pow}_{n}=1$ as long as $S^{2}(f, \mathbf{z}, F(x))>0$.

Proof. First of all, it is easy to see that

$$
\begin{aligned}
\text { Pow }_{n} & =P\left\{F\left(p, n-2 p, n R_{n}\right)>f_{\alpha}(p, n-2 p)\right\} \\
& =P\left\{\frac{X_{n} / p}{Y_{n} /(n-2 p)}>f_{\alpha}(p, n-2 p)\right\},
\end{aligned}
$$

where $X_{n}=\mathcal{X}^{2}\left(p, n R_{n}\right), Y_{n}=\mathcal{X}^{2}(n-2 p)$ are independent.

Because $\lim _{n \rightarrow \infty} n R_{n}=\infty$, then $\lim _{n \rightarrow \infty} \frac{X_{n}}{p}=\infty$ (a.s). 
Moreover, $\lim _{n \rightarrow \infty} \frac{Y_{n}}{n-2 p}=1($ a.s $)$ and $\lim _{n \rightarrow \infty} f_{\alpha}(p, n-2 p)=\frac{1}{p} \mathcal{X}_{\alpha}^{2}(p)$, then the last probability converges to 1 that yields 1$)$.

Now, according to Theorem 1 ,

$$
\lim _{n \rightarrow \infty} R_{n}=\lim _{n \rightarrow \infty} \frac{S^{2}\left(f, \mathbf{z}, F_{n}(x)\right)}{\sigma^{2}}=\frac{S^{2}(f, \mathbf{z}, F(x))}{\sigma^{2}}=R>0,
$$

then 2) is implied straight from 1).

\section{References}

[1] Aue, A., Horvath, L., Huskova, M. and Kokoszka, P. (2008) Testing for Changes in Polynomial Regression. Bernoulli, 14, 637-660. http://dx.doi.org/10.3150/08-BEJ122

[2] Berkes, I., Horvath, L. and Schauer, J. (2011) Asymptotics of Trimmed CUSUM Statistics. Bernoulli, 17, 1344-1367. http://dx.doi.org/10.3150/10-BEJ318

[3] Worsley, K.J. (1983) Testing for a Two-Phase Multiple Regression. Technometrics, 25, 35-42. http://dx.doi.org/10.1080/00401706.1983.10487817

[4] Koul, H.L. and Qian, L. (2002) Asymptotics of Maximum Likelihood Estimator in a Two-Phase Linear Regression Model. Journal of Statistical Planning and Inference, 108, 99-119. http://dx.doi.org/10.1016/S0378-3758(02)00273-2

[5] Jaruskova, D. (1998) Testing Appearance of Linear Trend. Journal of Statistical Planning and Inference, 70, 263-276. http://dx.doi.org/10.1016/S0378-3758(97)00184-5

[6] Lehmann, E.L. and Romano, J.P. (2005) Testing Statistical Hypotheses. 3th Edition, Springer, New York, USA, 277282.

[7] Ban, T.V., Quyen, N.T. and Ha, P.T. (2013) The Roughness of the Model Function to the Basis Functions. Journal of Mathematics and System Science, 3, 385-390.

[8] Bischoff, W. and Miller, F. (2000) Asymptotically Optimal Test and Optimal Designs for Testing the Mean in Regression Models with Applications to Change-Point Problems. Annals of the Institute of Statistical Mathematics, 52, 658679. http://dx.doi.org/10.1023/A:1017521225616 
Scientific Research Publishing (SCIRP) is one of the largest Open Access journal publishers. It is currently publishing more than 200 open access, online, peer-reviewed journals covering a wide range of academic disciplines. SCIRP serves the worldwide academic communities and contributes to the progress and application of science with its publication.

Other selected journals from SCIRP are listed as below. Submit your manuscript to us via either submit@scirp.org or Online Submission Portal.
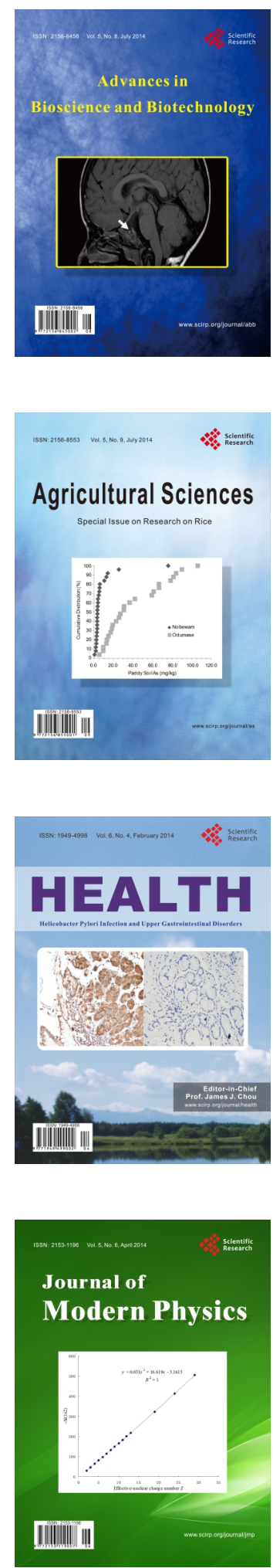
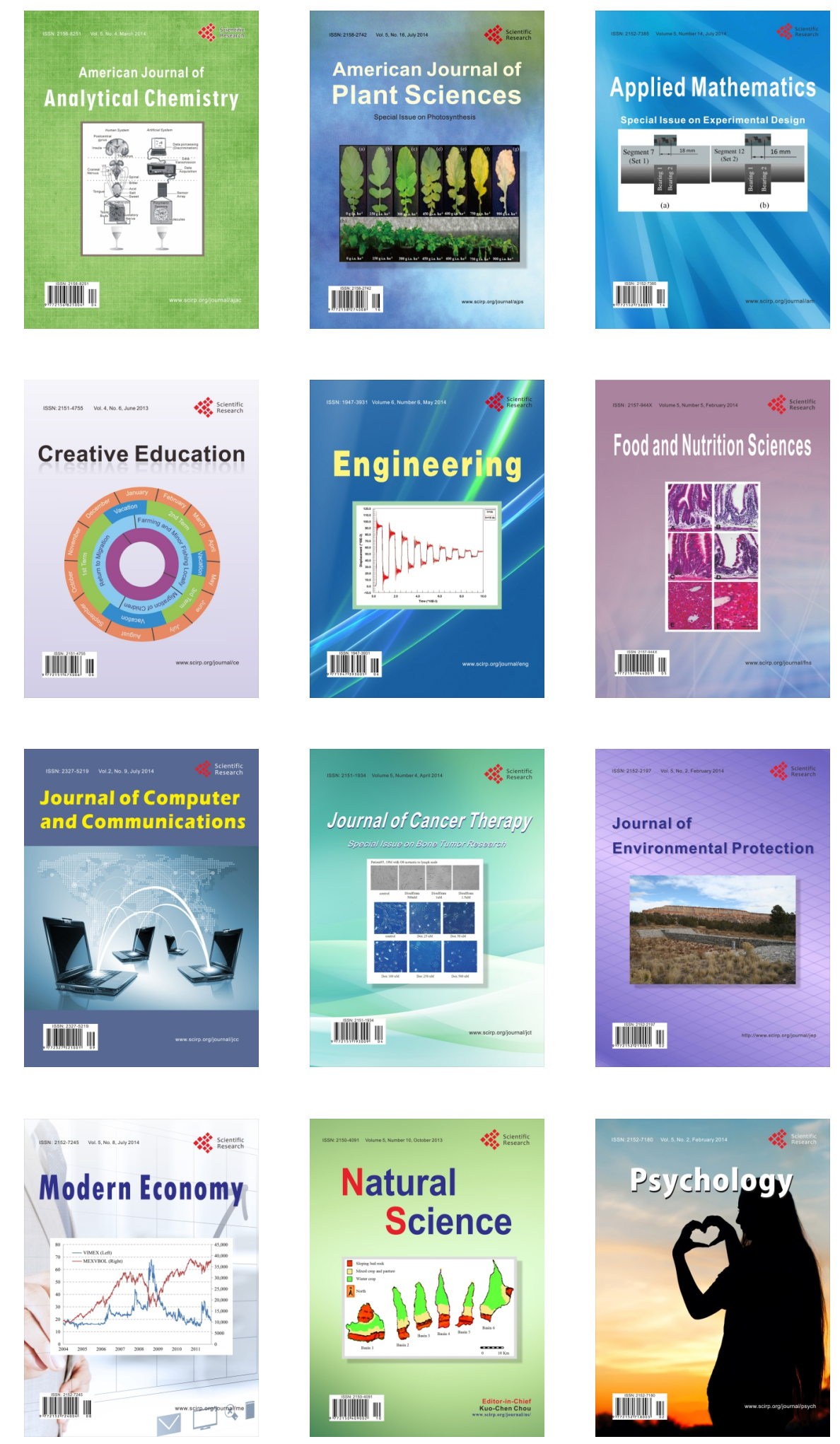